\title{
Impacts of Glycemic Control on Intracranial Plaque in Patients with Type 2 Diabetes Mellitus: A Vessel Wall MRI Study
}

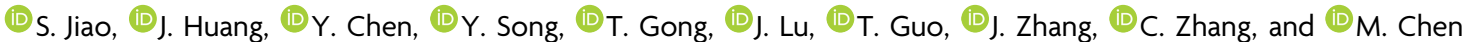

\begin{abstract}
BACKGROUND AND PURPOSE: The relationship between glycemic control in patients with type 2 diabetes mellitus and intracranial atherosclerotic plaque features has remained understudied. This study aimed to investigate the association of type 2 diabetes mellitus and glycemic control with the characteristics of intracranial plaques using vessel wall MR imaging.
\end{abstract}

MATERIALS AND METHODS: In total, 311 patients (217 [69.8\%] men; mean age, $63.24 \pm 11.44$ years) with intracranial atherosclerotic plaques detected on vessel wall MR imaging were enrolled and divided into 3 groups according to type 2 diabetes mellitus and glycemic control statuses: the non-type 2 diabetes mellitus group, the type 2 diabetes mellitus with good glycemic control group, and the type 2 diabetes mellitus with poor glycemic control group. The imaging features of intracranial plaque were analyzed and compared among the groups. The clinical risk factors for atherosclerosis were also analyzed using logistic regression analysis.

RESULTS: The plaque length and thickness were significantly higher in the type 2 diabetes mellitus with poor glycemic control group than in the non-type 2 diabetes mellitus group. The prevalence of strongly enhanced plaques was significantly higher in the type 2 diabetes mellitus with poor glycemic control group than in the non-type 2 diabetes mellitus and type 2 diabetes mellitus with good glycemic control groups $(92.9 \%, 63.4 \%$, and $72.7 \%$, respectively; $P<.001)$. Multivariate logistic regression analysis showed a significant association of poor glycemic control with the plaque length $(\mathrm{OR}=1.966 ; 95 \% \mathrm{Cl}, 1.170-3.303 ; P=.011)$, plaque thickness $(\mathrm{OR}=1.981 ; 95 \% \mathrm{Cl}, 1.174-3.340 ; P=.010)$, and strongly enhanced plaque (OR=5.448; 95\% $\mathrm{Cl}, 2.385-12.444 ; P<.001)$.

CONCLUSIONS: Poor glycemic control, compared with the history of diabetes, might have a greater impact on the burden and vulnerability of intracranial atherosclerotic plaques.

ABBREVIATIONS: ICAS = intracranial atherosclerosis; HbAlc = hemoglobin Alc; NDM = non-T2DM; T2DM $=$ type 2 diabetes mellitus; VW = vessel wall

$\mathbf{T}$ ype 2 diabetes mellitus (T2DM) is a highly prevalent disease associated with an increased risk of coronary artery disease, peripheral artery disease, and cerebrovascular disease, which are major causes of mortality. ${ }^{1}$ Diabetes alters the function of multiple cell types, including the endothelium, smooth muscle cells, and platelets, thus contributing to atherosclerosis and its complications. ${ }^{2-4}$ Diabetes also increases the breakdown and decreases

Received June 16, 2020; accepted after revision August 27.

From the Departments of Radiology (S.J., J.H., Y.S., T. Guo, J.Z., C.Z., M.C.),

Neurology (Y.C., T.Gong), and Neurosurgery (J.L.), Beijing Hospital, National Center of Gerontology, Institute of Geriatric Medicine, Chinese Academy of Medical Sciences, P.R. China, Beijing, China.

This study was supported by the Capital's Funds for Health Improvement and Research, grant No. CFH 2020-2-4052.

Please address correspondence to Yan Song, MD, Department of Radiology, Beijing Hospital, National Center of Gerontology, Institute of Geriatric Medicine, Chinese Academy of Medical Sciences, P.R. China. No. 1 Dahua Rd, Dong Dan, Beijing, 100730, P.R. China; e-mail: firesong@sina.com

- Indicates open access to non-subscribers at www.ajnr.org

http://dx.doi.org/10.3174/ajnr.A6878 the synthesis of collagen, so that the stability of the plaque fibrous cap may decrease and the plaques may rupture more readily. ${ }^{4}$

The association between glycemic control and extracranial atherosclerosis in patients with T2DM has been extensively investigated. $^{5-7}$ Patients with T2DM or poor glycemic control have a predisposition to a higher burden and vulnerability of extracranial atherosclerotic disease. Compared with the extracranial arteries, intracranial arteries exhibit different histologic features, including denser internal elastic lamina, thinner media, less abundant adventitia, only a few elastic fibers, without an external elastic lamina. ${ }^{8,9}$ These unique histologic structures of the intracranial arteries may lead to different characteristics of intracranial atherosclerosis (ICAS) compared with extracranial atherosclerosis. However, few studies have analyzed the association between glycemic control and the properties of intracranial atherosclerotic plaques in patients with T2DM.

In recent years, high-resolution vessel wall (VW) MR imaging has been used to demonstrate the characteristics of intracranial 
plaques, including plaque morphology, plaque components, and inflammation. ${ }^{10}$ In this study, the association between glycemic control and characteristics of intracranial atherosclerotic plaques in patients with T2DM was investigated by imaging plaques with VW MR imaging. The risk factors for the heavy burden and vulnerability of intracranial atherosclerotic plaques in patients with T2DM were also investigated. The findings of this study provided novel insights into the role of glycemic control status of patients with T2DM in the progression of ICAS, which, in turn, provided necessary information to educate patients about the importance of glycemic control.

\section{MATERIALS AND METHODS \\ Patients}

The records of patients with cerebrovascular symptoms who underwent VW MR imaging between December 2017 and July 2019 were retrospectively reviewed. The inclusion criteria were as follows: 1) VW MR imaging performed within 2 weeks of symptom onset; and 2) at least 1 intracranial atherosclerotic plaque identified on VW MR imaging. The exclusion criteria were as follows: 1) nonatherosclerotic intracranial artery stenosis diseases, such as Moyamoya disease, artery dissection, or vasculitis; 2) autoimmune diseases or systemic/local infectious diseases; 3 ) extracranial carotid artery stenosis $\geq 50 \%$; 4) evidence of cardiac sources of emboli; 5) incomplete clinical record; and 6) poor image quality. This study was approved by the ethics committee of Beijing Hospital and was performed in accordance with the 1964 Declaration of Helsinki and its later amendments or comparable ethical standards. Informed consent of patients for this retrospective study was waived.

We obtained the following data on clinical characteristics from electronic medical records: age, sex, body mass index, smoking status (current smokers or time interval since abstinence being $<5$ years), hypertension (systolic blood pressure $\geq 140 \mathrm{~mm} \mathrm{Hg}$ and/or diastolic blood pressure $\geq 90 \mathrm{~mm} \mathrm{Hg}$ or current use of antihypertensive agents), grade of hypertension (grade 1, systolic blood pressure $=140-159 \mathrm{~mm} \mathrm{Hg}$ and/or diastolic blood pressure $=90-$ $99 \mathrm{~mm} \mathrm{Hg}$; and grade 2, systolic blood pressure $\geq 160 \mathrm{~mm} \mathrm{Hg}$ and/ or diastolic blood pressure $\geq 100 \mathrm{~mm} \mathrm{Hg}$ ), blood pressure uncontrolled (systolic blood pressure $\geq 140 \mathrm{~mm} \mathrm{Hg}$ and/or diastolic blood pressure $\geq 90 \mathrm{~mm} \mathrm{Hg}$ after treatment), T2DM (fasting glucose $\geq 7.0 \mathrm{mmol} / \mathrm{L}$, random glucose $\geq 11.1 \mathrm{mmol} / \mathrm{L}$, or hemoglobin Alc $[\mathrm{HbAlc}] \geq 7 \%$, or use of medication for glycemic control), HbAlc, hyperlipidemia (total cholesterol $\geq 5.18 \mathrm{mmol} / \mathrm{L}$, triglycerides $\geq 1.7 \mathrm{mmol} / \mathrm{L}$, low-density lipoprotein cholesterol $\geq 3.37 \mathrm{mmol} / \mathrm{L}$, high-density lipoprotein cholesterol $<1.04 \mathrm{mmol} /$ L, or use of lipid-lowering medication), total cholesterol, low-density lipoprotein cholesterol, high-density lipoprotein cholesterol, triglycerides, low-density lipoprotein cholesterol/high-density lipoprotein cholesterol ratio, history of coronary artery disease, and a family history of cardiovascular disease.

Good glycemic control was defined as an HbAlc level of $<7.0 \%$, and poor glycemic control was defined as an HbA1c level of $\geq 7.0 \%$. ${ }^{11}$ All enrolled patients were categorized into one of the following 3 groups according to the T2DM history and HbAlc level: 1) NDM group: patients without T2DM; 2) the T2DM with good glycemic control group; and 3) the T2DM with poor glycemic control group.

\section{MR Imaging Protocol}

All patients underwent MR imaging using an Achieva TX 3T MR imaging scanner (Philips Healthcare) with a 16-channel neurovascular coil. VW MR images were acquired using a T1-weighted sequence (volume isotropic turbo spin-echo acquisition) before and after contrast agent injection using the following parameters: $\mathrm{TR}=800 \mathrm{~ms}, \mathrm{TE}=18 \mathrm{~ms}, \mathrm{FOV}=200 \times 180 \times 40 \mathrm{~mm}^{3}$, voxel size $=0.6 \times 0.6 \times 0.6 \mathrm{~mm}^{3}$, and acquisition time $=6$ minutes 28 seconds. Postcontrast T1WI was performed 5 minutes after the injection of a single-dose $(0.1=\mathrm{mmol} / \mathrm{kg}$ of body weight $)$ gadolinium-based contrast agent (gadopentetate dimeglumine, Magnevist; Bayer HealthCare Pharmaceuticals). Imaging parameters for TOF-MRA were as follows: $\mathrm{TR}=25 \mathrm{~ms}$, TE $=3.45 \mathrm{~ms}$, FOV $=180 \times 180 \mathrm{~mm}^{2}$, voxel size $=0.55 \times 0.55 \times 1.1 \mathrm{~mm}^{3}$, and acquisition time $=3$ minutes 34 seconds.

\section{Image Analysis}

All the VW MR images were transferred to a PACS workstation. The image quality was evaluated using a 3-point scale: grade 0 , outer boundary of the artery and lumen not identifiable; grade I, outer boundary and/or lumen is partially obscured; and grade II, wall architecture depicted in detail and lumen and outer boundary clearly defined. A senior neuroradiologist with 12 years of experience (S.J.) assessed all the VW MR images. Only patients with grade II image quality were enrolled in this study. Because of the small size of the intracranial artery, the analysis of the plaque mainly focused on the proximal arteries, including cavernous (C4) to communicating (C7) segments of the internal carotid artery, A1 and A2 segments of the anterior cerebral artery, M1 and M2 segments of the middle cerebral artery, the basilar artery, V4 segment of the vertebral arteries, and P1 and P2 segments of the posterior cerebral artery.

The plaque length, plaque thickness, strength of plaque enhancement, and degree of luminal stenosis were analyzed with the following steps: With the multiplanar reformations tool in the PACS, the T1-weighted images were reconstructed in both long and short axes according to the orientation of the vessels at the site of the maximum stenosis. Intracranial atherosclerotic plaque was defined as eccentric wall thickening with or without luminal stenosis identified on both the reconstructed pre- and postcontrast T1-weighted images. The plaque length, plaque thickness, and luminal stenosis were measured 3 times by a senior neuroradiologist with 12 years of experience (S.J.) who was blinded to the clinical information at the site of the most stenotic lesion on reconstructed postcontrast T1-weighted images of each patient, and the values were then averaged. The degree of luminal stenosis was evaluated according to the Warfarin-Aspirin Symptomatic Intracranial Disease Study. ${ }^{12}$ Severe stenosis was defined as the degree of luminal stenosis of $\geq 70 \%$. The strength of plaque enhancement was compared with that of the pituitary parenchyma and was determined qualitatively on the postcontrast T1weighted images as strong or not strong. If the plaque enhancement was equal to the pituitary enhancement, it was deemed strong; if the enhancement was less than the pituitary 


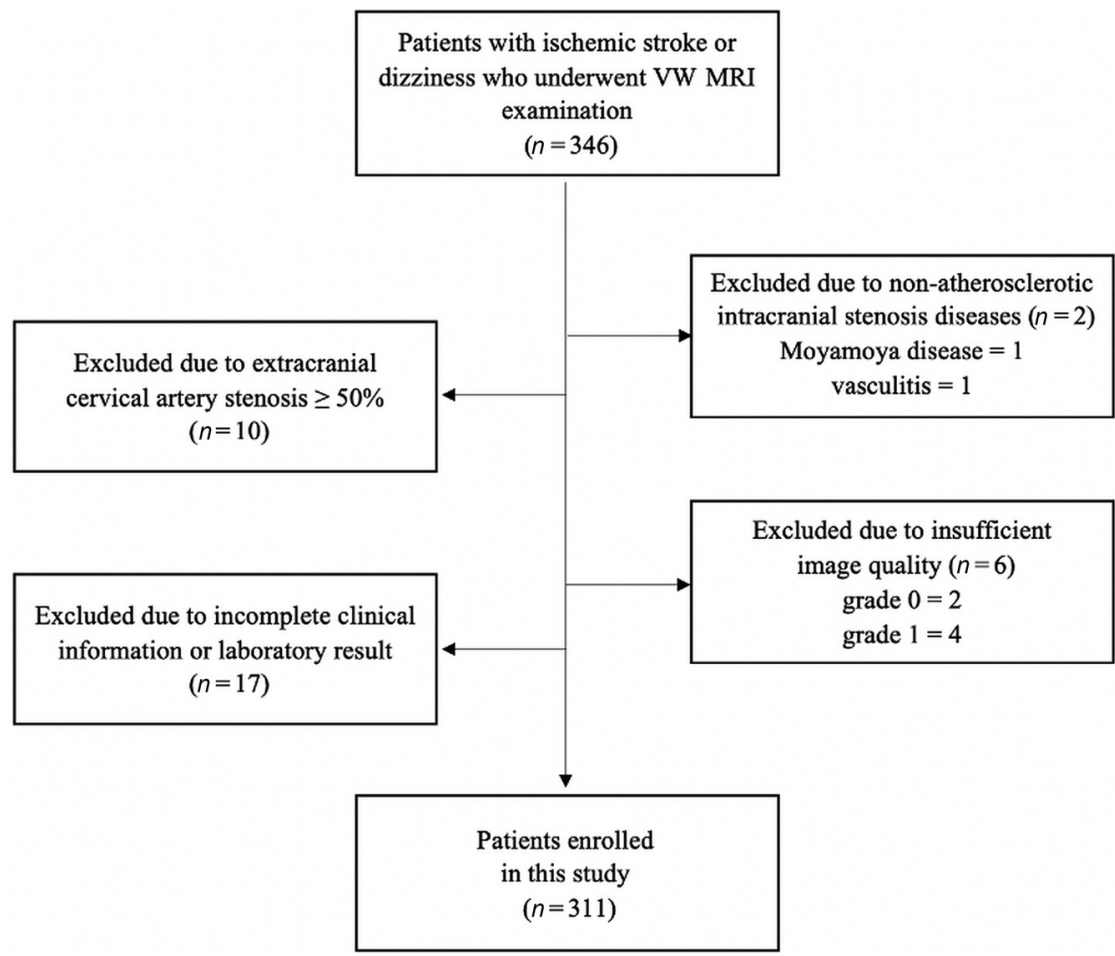

FIG 1. Flow diagram of study identification.

enhancement or showed no change compared with the precontrast images, it was deemed not strong. ${ }^{13}$ The strength of plaque enhancement was independently determined by 2 experienced neuroradiologists (S.J. and J.H.) with $>10$ years of experience who were blinded to the clinical data, and all disagreements were resolved by consensus.

\section{Statistical Analysis}

All continuous variables conforming to normal distribution were expressed as means $\pm S D$, the continuous variables with nonnormal distribution were described as median (25th-75th percentiles), and categoric variables were summarized as count and percentage. The characteristics of plaques were compared among the 3 groups using the 1-way ANOVA or Kruskal-Wallis test for continuous variables as appropriate and the $\chi^{2}$ test for categoric variables. Univariate and multivariate logistic regression analyses were performed to determine the independent risk factors for the heavy burden and vulnerability of intracranial atherosclerotic plaques. The plaque length, thickness at the median value, and luminal stenosis degree at $70 \%$ were dichotomized to investigate the risk factors for the heavy burden (plaque length, plaque thickness, and luminal stenosis degree) and vulnerability (strong enhancement) of intracranial atherosclerotic plaques.

The clinical data were also dichotomized for statistical analysis, including age (65 years or older versus younger than 65 years), sex (male), hypertension ( $\geq 140 / 90 \mathrm{~mm} \mathrm{Hg}$ versus $<140 / 90 \mathrm{~mm}$ $\mathrm{Hg}$ ), HbAlc ( $\geq 7 \%$ versus $<7 \%$ ), high total cholesterol level ( $\geq 5.18 \mathrm{mmol} / \mathrm{L})$, high triglyceride level $(\geq 1.7 \mathrm{mmol} / \mathrm{L})$, high lowdensity lipoprotein cholesterol $(\geq 3.37 \mathrm{mmol} / \mathrm{L})$, and low highdensity lipoprotein cholesterol level $(<1.04 \mathrm{mmol} / \mathrm{L})$. Intrareader agreement in the measurement of plaque burden was performed by intraclass correlation coefficient analysis, and interreader agreement in the identification of plaque enhancement was assessed by Cohen $\kappa$ analysis. All statistical analyses were performed using SPSS 25.0 (IBM). A $P$ value $<.05$ was considered statistically significant.

\section{RESULTS}

\section{Clinical Characteristics}

In total, 311 patients (217 [69.8\%] men; mean age, $63.24 \pm 11.44$ years) were enrolled in this study. A flow diagram summarizing the exclusion information is shown in Fig 1. Among all 311 patients, 281 (90.4\%) patients were diagnosed with ischemic stroke (162 patients positive for infarction on DWI, 119 patients with TIA), and the other $30(9.6 \%)$ patients had dizziness. Of the 311 patients, 139 (44.69\%) had T2DM and $172(55.31 \%)$ did not have T2DM. Of the 139 patients with T2DM, 55 (39.57\%) had good glycemic control and $84(60.43 \%)$ had poor glycemic control. The duration of T2DM was from 2 months to 30 years, with an average duration of 8.5 years, 95 (68.35\%) patients took oral medication, $32(23.02 \%)$ patients were on insulin therapy, and 12 (8.63\%) patients did not have any regular treatment. The clinical characteristics of the NDM, T2DM with good glycemic control, and T2DM with poor glycemic control groups are presented in Table 1. Compared with the NDM group, the T2DM with poor glycemic control group had a significantly lower high-density lipoprotein cholesterol level (0.92 mmol/L [interquartile range, $0.76-1.09 \mathrm{mmol} / \mathrm{L}$ ] versus $1.00 \mathrm{mmol} / \mathrm{L}$ [interquartile range, $0.89-1.15 \mathrm{mmol} / \mathrm{L}$ ], $P<.05)$. No significant difference was found in the proportion of patients with hypertension, the grade of hypertension, or patients with uncontrolled blood pressure after treatment among the 3 groups. No significant differences were observed among the groups for other clinical parameters.

\section{Plaque Characteristics among NDM, T2DM with Good Glycemic Control, and T2DM with Poor Glycemic Control Groups}

The mean plaque length and thickness in all 311 patients were 6.72 and $1.80 \mathrm{~mm}$, respectively. The plaques were significantly longer (6.45 mm [interquartile range, $4.63-10.95 \mathrm{~mm}$ ] versus $4.90 \mathrm{~mm}$ [interquartile range, $3.43-7.58 \mathrm{~mm}$ ], $P<.001)$ and thicker $(1.80 \mathrm{~mm}$ [interquartile range, $1.40-2.38 \mathrm{~mm}$ ] versus $1.40 \mathrm{~mm}$ [interquartile range, $1.10-2.18 \mathrm{~mm}$ ], $P=.005)$, and the luminal stenosis was significantly greater (66.67\% [interquartile range, $34.47 \%-80.15 \%$ ] versus $38.52 \%$ [interquartile range, $16.67 \%-72.67 \%$ ], $P<.001$ ) in the T2DM with poor glycemic control group than in the NDM group.

Of the 311 patients, plaques in 227 patients (72.99\%) were strongly enhanced. The prevalence of strongly enhanced plaques 


\begin{tabular}{|c|c|c|c|c|c|}
\hline & NDM Group $(n=172)$ & DMGGC Group ( $n=55)$ & DMPGC Group $(n=84)$ & $\chi^{2} / F$ & $P$ Value \\
\hline Male (No.) (\%) & 117 (68.02) & $44(80)$ & $56(66.67)$ & 3.362 & .186 \\
\hline Age (yr) & $62.44 \pm 11.87$ & $63.71 \pm 9.90$ & $64.60 \pm 11.47$ & 1.061 & .347 \\
\hline $\mathrm{BMI}\left(\mathrm{Kg} / \mathrm{m}^{2}\right)$ & $25.66 \pm 3.37$ & $25.60 \pm 3.00$ & $25.50 \pm 3.37$ & 0.066 & .936 \\
\hline Smoking (No.) (\%) & $73(42.44)$ & $19(34.55)$ & $36(42.86)$ & 1.210 & .546 \\
\hline Hypertension (No.) (\%) & $129(75)$ & $48(87.28)$ & $69(82.14)$ & 4.441 & .109 \\
\hline Grade 1 hypertension (No.) (\%) & $90(69.77)$ & 37 (77.08) & $52(75.36)$ & 1.271 & .530 \\
\hline Grade 2 hypertension (No.) (\%) & $39(30.23)$ & $11(22.92)$ & $17(24.64)$ & & \\
\hline BP uncontrolled (No.) (\%) & $84(65.12)$ & $36(75)$ & $50(72.5)$ & 2.107 & .349 \\
\hline History of CAD (No.) (\%) & $25(14.53)$ & $15(27.27)$ & $17(20.24)$ & 4.798 & .091 \\
\hline Family history of CVD (No.) (\%) & $22(12.79)$ & $5(9.09)$ & 9 (10.71) & 0.641 & .726 \\
\hline Hyperlipemia (No.) (\%) & $111(64.53)$ & $37(67.27)$ & $47(55.95)$ & 2.375 & .305 \\
\hline Total cholesterol (mmol/L) & $3.81(3.10-4.70)$ & $3.24(2.94-4.48)$ & $3.35(2.96-4.22)$ & 5.946 & .051 \\
\hline $\mathrm{LDL}(\mathrm{mmol} / \mathrm{L})$ & $2.22(1.67-2.93)$ & $1.81(1.48-2.81)$ & $2.07(1.58-2.63)$ & 4.918 & .086 \\
\hline $\mathrm{HDL}(\mathrm{mmol} / \mathrm{L})$ & $1.00(0.89-1.15)^{b}$ & $0.99(0.87-1.19)$ & $0.92(0.76-1.09)^{b}$ & 9.796 & .008 \\
\hline Triglycerides (mmol/L) & 1.37 (0.97-1.93) & $1.16(0.90-1.82)$ & $1.32(0.96-1.82)$ & 1.749 & .417 \\
\hline LDL/HDL ratio & $2.12(1.64-2.93)$ & $1.81(1.53-2.40)$ & $2.21(1.77-2.87)$ & 4.565 & .102 \\
\hline
\end{tabular}

Note:-DMGGC indicates T2DM with good glycemic control; DMPGC, T2DM with poor glycemic control; BMI, body mass index; LDL, low-density lipoprotein cholesterol; $\mathrm{HDL}$, high-density lipoprotein cholesterol; CAD, coronary artery disease; CVD, cardiovascular disease; BP, blood pressure.

${ }^{a}$ Continuous variables with normal distribution are presented as means \pm SD; continuous variables with non-normal distribution are presented as median (25th-75th percentiles); and categoric variables are presented as (No.) (\%).

${ }^{\mathrm{b}} \mathrm{P}$ value $<.05$.

Table 2: The characteristics of intracranial plaque among NDM, DMGGC, and DMPGC groups ${ }^{\mathrm{a}}$

\begin{tabular}{lccrrr}
\hline & NDM Group $(\boldsymbol{n}=172)$ & DMGGC Group $(\boldsymbol{n}=\mathbf{5 5})$ & DMPGC Group $(\boldsymbol{n}=84)$ & $\boldsymbol{\chi}^{2}$ & $\boldsymbol{P}$ Value \\
\hline Plaque length (mm) & $4.90(3.43-7.58)^{\mathrm{b}}$ & $6.10(3.70-8.00)$ & $6.45(4.63-10.95)^{\mathrm{b}}$ & 19.086 & $<.001$ \\
Plaque thickness (mm) & $1.40(1.10-2.18)^{\mathrm{b}}$ & $1.60(1.20-2.20)$ & $1.80(1.40-2.38)^{\mathrm{b}}$ & 10.043 & .005 \\
Lumen stenosis (\%) & $38.52(16.67-72.67)^{\mathrm{b}}$ & $54.83(39.29-72.41)$ & $66.67(34.47-80.15)^{\mathrm{b}}$ & 17.757 & $<.001$ \\
Strong enhancement (No.) (\%) & $109(63.37)$ & $40(72.73)$ & & $78(92.86)^{\mathrm{b}}$ & 2.617 \\
& $109(63.37)^{\mathrm{b}}$ & & .203 & 24.921 & $<.001$ \\
& & $40(72.73)^{\mathrm{b}}$ & $78(92.86)^{\mathrm{b}}$ & 10.501 & .001 \\
\hline
\end{tabular}

Note:-DMGGC indicates T2DM with good glycemic control; DMPGC, T2DM with poor glycemic control.

${ }^{a}$ Continuous variables with non-normal distribution are presented as median (25th-75th percentiles); categoric variables are presented as (No.) (\%).

${ }^{\mathrm{b}} \mathrm{P}$ value $<.05$

was significantly higher in the T2DM with poor glycemic control group (92.9\%) than in the NDM $(63.4 \%)$ and T2DM with good glycemic control $(72.7 \%)$ groups $(P<.001)$. Although the prevalence of strongly enhanced plaques was higher in the T2DM with good glycemic control group than in the NDM group, no significant difference was observed.

The characteristics of intracranial plaques (plaque length, plaque thickness, luminal stenosis, and plaque enhancement) among the NDM, T2DM with good glycemic control, and T2DM with poor glycemic control groups are presented in Table 2 . The representative cases with strongly enhanced plaque are presented in Fig 2.

\section{Risk Factors for the Heavy Burden and Vulnerability of Intracranial Atherosclerotic Plaques}

In univariate logistic regression analysis, male sex and poor glycemic control were significantly associated with plaque length $(\mathrm{OR}=$ 1.789; 95\% CI, 1.095-2.924; $P=.020$; and $\mathrm{OR}=1.888,95 \% \mathrm{CI}$, $1.132-3.150 ; P=.015$, respectively), low high-density lipoprotein cholesterol and poor glycemic control were significantly associated with plaque thickness $(\mathrm{OR}=1.833$; 95\% $\mathrm{CI}, 1.168-2.876 ; P=.008$; and $\mathrm{OR}=2.091 ; 95 \% \mathrm{CI}, 1.247-3.50 ; P=.005$, respectively), poor glycemic control was significantly associated with severe luminal stenosis (OR $=1.962 ; 95 \% \mathrm{CI}, 1.169-3.294 ; P=.011)$, and low high-density lipoprotein cholesterol and poor glycemic control were significantly associated with strong enhancement of intracranial plaques $(\mathrm{OR}=2.213 ; 95 \% \mathrm{CI}, 1.329-3.685 ; P=.002$; and $\mathrm{OR}=$ 5.758; 95\% CI, 2.534-13.085; $P<.001$, respectively).

The multivariate logistic regression analysis showed that poor glycemic control was an independent risk factor for plaque length, plaque thickness, severe luminal stenosis, and strong enhancement of intracranial plaque $(\mathrm{OR}=1.966 ; 95 \% \mathrm{CI}, 1.170-3.303 ; P=.011$; $\mathrm{OR}=1.981,95 \% \mathrm{CI}, 1.174-3.340 ; P=.010 ; \mathrm{OR}=1.962 ; 95 \% \mathrm{CI}$, 1.169-3.294; $P=.011$; and $\mathrm{OR}=5.448$; 95\% CI, 2.385-12.444; $P<.001$ for plaque length, plaque thickness, severe luminal stenosis, and strong enhancement, respectively) after adjustment for age, hypertension, smoking, history of coronary heart disease, and family history of cardiovascular disease. In addition, male sex was significantly associated with plaque length $(\mathrm{OR}=1.864 ; 95 \% \mathrm{CI}$, 1.132-3.068; $P=.014$ ), and low high-density lipoprotein cholesterol was significantly associated with plaque thickness $(\mathrm{OR}=$ $1.739,95 \% \mathrm{CI}, 1.102-2.745 ; P=.017)$ and strong enhancement of intracranial plaques $(\mathrm{OR}=2.046 ; 95 \% \mathrm{CI}, 1.208-3.465 ; P=.008)$. The results of univariate and multivariate logistic regression analyses are presented in Table 3.

\section{Intraobserver and Interobserver Reliability for Measurement}

The intraobserver reliability was good for the measurement of plaque length (intraclass correlation coefficient $=0.821 ; 95 \% \mathrm{CI}$, $0.645-0.931 ; P<.001$; plaque thickness (intraclass correlation 
coefficient $=0.846,95 \% \mathrm{CI}, 0.632-0.953 ; P<.001)$, and luminal stenosis (intraclass correlation coefficient $=0.973 ; 95 \% \mathrm{CI}$, 0.968-0.978; $P<.001$ ). The interobserver reliability was also high for the evaluation of the strength of plaque enhancement $(\kappa$ value $=0.856 ; 95 \%$ CI, 0.791-0.921; $P<.001)$.

\section{DISCUSSION}

The burden and vulnerability of intracranial atherosclerotic plaques are very important parameters when analyzing atherosclerosis due to their strong association with ischemic stroke. ${ }^{14-16}$ In the present study, these intracranial plaque features were compared among patients with different diabetes and glycemic control statuses. The patients with T2DM having poor glycemic control tended to have a much heavier plaque burden and more vulnerable plaque. Poor glycemic control was an independent risk factor for intracranial plaque severity based on the multivariable logistic regression analysis. This finding suggested that the glycemic control status might have a greater impact than the history of diabetes on ICAS.
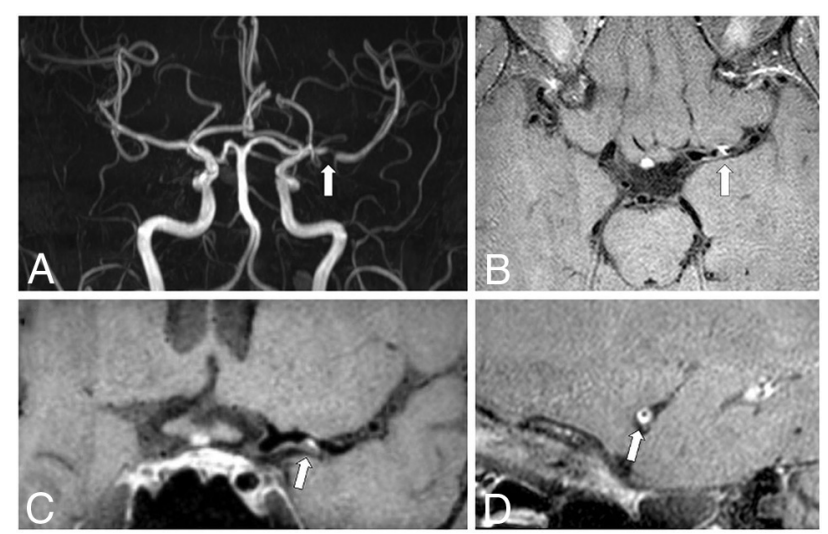

FIG 2. Strong enhancement of plaque in the MCA. A, TOF-MRA shows severe stenosis in the M1 segment of the left MCA (arrow). B, Postcontrast T1-weighted image (axial acquisition) shows wall thickening at the corresponding location (arrow). C, Reconstructed T7weighted image shows the length of plaque (arrow) in the long axis of the MCA at the site of the most stenotic lesion. $D$, Reconstructed T1-weighted image shows the thickness of plaque (arrow) in the short axis of the MCA at the site of the most stenotic lesion.
Previous studies showed that the plaque burden of the extracranial vessels was significantly heavier in patients with $\mathrm{T} 2 \mathrm{DM}$ or poor glycemic control than in those without T2DM or with good glycemic control. According to a meta-analysis of 23 studies, including 4019 patients with T2DM and 1110 patients with impaired glucose tolerance among 24,111 patients, the patients with T2DM and impaired glucose tolerance had greater carotid intima-media thickness than the control patients. The mean difference was $0.13 \mathrm{~mm}$ (95\% CI, 0.12-0.14 mm) and $0.04 \mathrm{~mm}$ (95\% CI, 0.014-0.071 mm), respectively. ${ }^{17}$ The parameters of carotid plaque burden, such as percentage of luminal stenosis, maximum wall thickness, and percentage wall volume, were significantly greater in patients with hypertension with a high HbAlc than in those with a low HbA1c. ${ }^{18}$

The results of the present study were consistent with those of previous studies on extracranial atherosclerosis. The intracranial plaque burden was significantly heavier in patients with T2DM and poor glycemic control than in those without T2DM. Longterm hyperglycemia has been recognized as a major factor in the pathogenesis of atherosclerosis. ${ }^{19}$ The results of the present study suggested that although the histologic structure of intracranial arteries was different from that of extracranial arteries, the progression of ICAS was affected by long-term hyperglycemia, consistent with the results on extracranial atherosclerosis. This finding might be because continuous exposure to hyperglycemia induced a series of alterations at the cellular level of vascular tissues, for example, overproduction of reactive oxygen species, increased formation of advanced glycation end-products, and activation of the advanced glycation end-product receptors for advanced glycation end-product axis, polyol and hexosamine flux, protein kinase $\mathrm{C}$ activation, and chronic vascular inflammation, ${ }^{20}$ which potentially promote accelerated atherosclerosis. However, no significant difference in the intracranial plaque burden was found between patients with T2DM and good glycemic control and those without T2DM. These findings indicated that the long-term glycemic control status might have a greater impact than the history of diabetes on the intracranial plaque burden, and the risk of heavy plaque burden in patients with T2DM and good glycemic control might not be higher than that in those without T2DM.

The plaque enhancement is related to the neovascularity within plaques and the increased endothelial permeability, which

Table 3: Association between risk factors of cardiovascular disease and heavy burden and vulnerability of intracranial plaques

\begin{tabular}{|c|c|c|c|c|c|c|}
\hline & \multicolumn{3}{|c|}{ Univariate Regression } & \multicolumn{3}{|c|}{ Multivariate Regression $^{\mathrm{a}}$} \\
\hline & OR & $95 \% \mathrm{Cl}$ & $P$ Value & OR & $95 \% \mathrm{Cl}$ & $P$ Value \\
\hline \multicolumn{7}{|l|}{ Plaque length } \\
\hline Male & 1.789 & $1.095-2.924$ & .020 & 1.864 & $1.132-3.068$ & .014 \\
\hline Poor glycemic control & 1.888 & $1.132-3.150$ & .015 & 1.966 & $1.170-3.303$ & .011 \\
\hline \multicolumn{7}{|l|}{ Plaque thickness } \\
\hline Low HDL & 1.833 & $1.168-2.876$ & .008 & 1.739 & $1.102-2.745$ & .017 \\
\hline Poor glycemic control & 2.091 & $1.247-3.507$ & .005 & 1.981 & $1.174-3.340$ & .010 \\
\hline \multicolumn{7}{|l|}{ Severe lumen stenosis } \\
\hline Poor glycemic control & 1.962 & $1.169-3.294$ & .011 & 1.962 & $1.169-3.294$ & .011 \\
\hline \multicolumn{7}{|l|}{ Strong enhancement } \\
\hline Low HDL & 2.213 & $1.329-3.685$ & .002 & 2.046 & $1.208-3.465$ & .008 \\
\hline Poor glycemic control & 5.758 & $2.534-13.085$ & $<.001$ & 5.448 & $2.385-12.444$ & $<.001$ \\
\hline
\end{tabular}

Note:-LDL indicates low-density lipoprotein cholesterol; HDL, high-density lipoprotein cholesterol.

${ }^{a}$ Multivariate logistic regression adjusted for age, smoking, hypertension, history of coronary artery disease, and family history of cardiovascular disease. 
facilitate the entry of contrast agents from the blood plasma. ${ }^{21-24}$ The strong enhancement of intracranial plaques was reported as an imaging marker of plaque vulnerability, which correlated with recent ischemic stroke. ${ }^{25-27}$ Moreno et $\mathrm{al}^{28}$ demonstrated that coronary artery plaques from patients with diabetes exhibited a larger content of lipid-rich atheroma and macrophage infiltration, suggesting an increased vulnerability compared with those from patients without diabetes. Gao et $\mathrm{al}^{29}$ reported that patients with diabetes had a significantly higher prevalence of high-risk carotid plaque $(29.7 \%$ versus $19.9 \%, P=.011)$ than those without diabetes. In this study, the intracranial plaque vulnerability was compared between different diabetes and glycemic control statuses; a higher prevalence of strongly enhanced plaques was found more often in patients with T2DM and poor glycemic control than in patients with T2DM and good glycemic control and patients without T2DM. This result might be due to the diabetic arterial endothelial dysfunction expressed by increased vascular permeability and vasa vasorum neovascularization related to hyperglycemia. ${ }^{2}$ Moreover, no significant difference in the prevalence of strongly enhanced plaques was found between patients without T2DM and those with T2DM and good glycemic control. These findings suggested that the poor glycemic control might have a greater impact than the history of T2DM on the plaque vulnerability, and the risk of plaque vulnerability in patients with T2DM and good glycemic control might not be higher than that in those without T2DM.

This study found an $\mathrm{HbAlc}$ value of $>7 \%$ to be an independent risk factor for the heavy burden and vulnerability of intracranial atherosclerotic plaques based on the multivariate logistic regression analysis. HbA1c was used as a serum biochemical index to estimate the long-term glycemic control, which represented an average blood glucose during the preceding 2-3 months and tracked well in individuals across time. ${ }^{30}$ Only a limited number of studies have reported the relationship between glycemic control status and MR imaging morphologic and enhancement parameters of plaques in atherosclerosis. Mukai et $\mathrm{al}^{31}$ indicated that the multivariable-adjusted odds ratios of the presence of carotid wall thickening significantly increased with elevated HbAlc levels. Sun et $\mathrm{al}^{18}$ reported a positive association between $\mathrm{HbAlc}$ and the presence of a lipid-rich necrotic core in carotid arterial plaques on MR imaging. However, few previous studies showed the impact of poor glycemic control in patients with T2DM on the heavy burden and vulnerability of intracranial atherosclerotic plaques. A recent study by Choi et $\mathrm{al}^{32}$ showed that poor glycemic control was associated with multiple intracranial stenoses, reflecting the extent and severity of ICAS. The results of the present study were consistent with the findings of Choi et al from a totally new point of view.

The present study has several limitations. First, only the length and thickness of plaques and the prevalence of strongly enhanced plaques were measured. In a future study, the volume of plaque and the degree of plaque enhancement should be measured to quantitatively evaluate the burden and vulnerability of intracranial atherosclerotic plaques more precisely. Second, only the large-tomiddle-sized intracranial arteries were assessed in this study due to the small size of the intracranial artery and the limit of the spatial resolution of VW MR imaging. Further investigation on small intracranial arteries should be performed with the improvement in MR imaging. Finally, this was a retrospective cross-sectional study.
In the future, prospective longitudinal studies should be conducted to further estimate the changes of intracranial plaques after treating poor glucose control.

\section{CONCLUSIONS}

This study showed that the burden and vulnerability of intracranial atherosclerotic plaques were significantly greater in patients with T2DM and poor glycemic control than in those without T2DM, while no significant difference was found between patients without T2DM and those with T2DM and good glycemic control. Poor glycemic control might have a greater impact than the history of diabetes on the burden and vulnerability of intracranial plaques.

Disclosures: Yan Song - RELATED: Grant: Capital's Funds for Health Improvement and Research, Comments: We received this grant with the research topic: Constructing the risk model for predicting acute stroke recurrence: a study with intra- and extracranial artery one-stop vessel wall imaging and artificial intelligence image analysis.

\section{REFERENCES}

1. Christen AI, Armentano RL, Miranda A, et al. Arterial wall structure and dynamics in type 2 diabetes mellitus methodological aspects and pathophysiological findings. Curr Diabetes Rev 2010;6:367-77 CrossRef Medline

2. Moreno PR, Fuster V. New aspects in the pathogenesis of diabetic atherothrombosis. J Am Coll Cardiol 2004;44:2293-2300 CrossRef Medline

3. Creager MA, Lüscher TF, Cosentino F, et al. Diabetes and vascular disease: pathophysiology, clinical consequences, and medical therapy, Part I. Circulation 2003;108:1527-32 CrossRef Medline

4. Beckman JA, Creager MA, Libby P. Diabetes and atherosclerosis: epidemiology, pathophysiology, and management. JAMA 2002;287:257081 CrossRef Medline

5. Selvin E, Coresh J, Golden SH, et al; Atherosclerosis Risk in Communities Study. Glycemic control, atherosclerosis, and risk factors for cardiovascular disease in individuals with diabetes: the Atherosclerosis Risk in Communities Study. Diabetes Care 2005;28:1965-73 CrossRef Medline

6. Yang DJ, Lee MS, Kim WH, et al. The impact of glucose control on coronary plaque composition in patients with diabetes mellitus. $J$ Invasive Cardiol 2013;25:137-41 Medline

7. Tavares CA, Rassi CH, Fahel MG, et al. Relationship between glycemic control and coronary artery disease severity, prevalence and plaque characteristics by computed tomography coronary angiography in asymptomatic type 2 diabetic patients. Int J Cardiovasc Imaging 2016;32:1577-85 CrossRef Medline

8. Moossy J. Morphology, sites and epidemiology of cerebral atherosclerosis. Res Publ Assoc Res Nerv Ment Dis 1966;41:1-22 Medline

9. Velican C. Studies on the age-related changes occurring in human cerebral arteries. Atherosclerosis 1970;11:509-29 CrossRef Medline

10. Ryu CW, Kwak HS, Jahng GH, et al. High-resolution MRI of intracranial atherosclerotic disease. Neurointervention 2014;9:9-20 CrossRef Medline

11. American Diabetes Association. Standards of Medical Care in Diabetes-2019 Abridged for Primary Care Providers. Clin Diabetes 2019;37:11-34 CrossRef Medline

12. Chimowitz MI, Kokkinos J, Strong J, et al. The Warfarin-Aspirin Symptomatic Intracranial Disease Study. Neurology 1995;45:148893 CrossRef Medline

13. Skarpathiotakis M, Mandell DM, Swartz RH, et al. Intracranial atherosclerotic plaque enhancement in patients with ischemic stroke. AJNR Am J Neuroradiol 2013;34:299-304 CrossRef Medline

14. Li F, Chen QX, Chen ZB, et al. Magnetic resonance imaging of plaque burden in vascular walls of the middle cerebral artery correlates with cerebral infarction. CNR 2016;13:263-70 CrossRef Medline 
15. Ryu CW, Jahng GH, Kim EJ, et al. High-resolution wall and lumen MRI of the middle cerebral arteries at 3 Tesla. Cerebrovasc Dis 2009;27:433-42 CrossRef Medline

16. Huang J, Jiao S, Zhao X, et al. Characteristics of patients with enhancing ICAS and association between plaque enhancement and recent cerebrovascular ischemic events: a high-resolution magnetic resonance imaging study. Acta Radiol 2019;60:1301-07 CrossRef Medline

17. Brohall G, Odén A, Fagerberg B. Carotid artery intima-media thickness in patients with type 2 diabetes mellitus and impaired glucose tolerance: a systematic review. Diabet Med 2006;23:609-16 CrossRef Medline

18. Sun B, Zhao H, Liu X, et al. Elevated hemoglobin Alc is associated with carotid plaque vulnerability: novel findings from magnetic resonance imaging study in hypertensive stroke patients. Sci Rep 2016;6:33246 CrossRef Medline

19. Aronson D, Rayfield EJ. How hyperglycemia promotes atherosclerosis: molecular mechanisms. Cardiovasc Diabetol 2002;1:1 CrossRef Medline

20. Katakami N. Mechanism of development of atherosclerosis and cardiovascular disease in diabetes mellitus. $J$ Atheroscler Thromb 2018;25:27-39 CrossRef Medline

21. O’Brien KD, Allen MD, McDonald TO, et al. Vascular cell adhesion molecule-1 is expressed in human coronary atherosclerotic plaques. implications for the mode of progression of advanced coronary atherosclerosis. J Clin Invest 1993;92:945-51 CrossRef Medline

22. O'Brien KD, McDonald TO, Chait A, et al. Neovascular expression of E-selectin, intercellular adhesion molecule-1, and vascular cell adhesion molecule-1 in human atherosclerosis and their relation to intimal leukocyte content. Circulation 1996;93:672-82 CrossRef Medline

23. Moulton KS, Vakili K, Zurakowski D, et al. Inhibition of plaque neovascularization reduces macrophage accumulation and progression of advanced atherosclerosis. Proc Natl Acad Sci U S A 2003;100:473641 CrossRef Medline

24. Celletti FL, Waugh JM, Amabile PG, et al. Vascular endothelial growth factor enhances atherosclerotic plaque progression. Nat Med 2001;7:425-29 CrossRef Medline

25. Lou X, Ma N, Ma L, et al. Contrast-enhanced 3T high-resolution MR imaging in symptomatic atherosclerotic basilar artery stenosis. AJNR Am J Neuroradiol 2013;34:513-17 CrossRef Medline

26. Ryu CW, Jahng GH, Shin HS. Gadolinium enhancement of atherosclerotic plaque in the middle cerebral artery: relation to symptoms and degree of stenosis. AJNR Am J Neuroradiol 2014;35:230610 CrossRef Medline

27. Qiao Y, Zeiler SR, Mirbagheri S, et al. Intracranial plaque enhancement in patients with cerebrovascular events on high-spatial-resolution MR images. Radiology 2014;271:534-42 CrossRef Medline

28. Moreno PR, Murcia AM, Palacios IF, et al. Coronary composition and macrophage infiltration in atherectomy specimens from patients with diabetes mellitus. Circulation 2000;102:2180-84 CrossRef Medline

29. Gao X, Song J, Watase H, et al; CARE-II Investigators. Differences in carotid plaques between symptomatic patients with and without diabetes mellitus. Arterioscler Thromb Vasc Biol 2019;39:1234-39 CrossRef Medline

30. Meigs JB, Nathan DM, Cupples LA, et al. Tracking of glycated hemoglobin in the original cohort of the Framingham Heart Study. $J$ Clin Epidemiol 1996;49:411-17 CrossRef Medline

31. Mukai N, Ninomiya T, Hata J, et al. Association of hemoglobin A1c and glycated albumin with carotid atherosclerosis in communitydwelling Japanese subjects: the Hisayama Study. Cardiovasc Diabetol 2015;14:24 CrossRef Medline

32. Choi N, Lee JY, Sunwoo JS, et al. Recently uncontrolled glycemia in diabetic patients is associated with the severity of intracranial atherosclerosis. J Stroke Cerebrovasc Dis 2017;26:2615-21 CrossRef Medline 